\title{
ATUAÇÃO DE ENFERMEIROS LÍDERES DE UNIDADE DE TERAPIA INTENSIVA: ABORDAGEM COMPREENSIVA
}

Claudete Aparecida Conz $z^{1}$

Reginaldo Santos de Aguiar ${ }^{2}$

Heliandra Holanda Reis ${ }^{2}$

Maria Cristina de Jesus Pinto ${ }^{3}$

Vera Lúcia Mira ${ }^{1}$

Miriam Aparecida Barbosa Merighi ${ }^{1}$
https://orcid.org/0000-0002-1204-185X

https://orcid.org/0000-0002-4685-2423

https://orcid.org/0000-0002-0964-4238

https://orcid.org/0000-0002-8854-690X

https://orcid.org/0000-0002-5896-3948

https://orcid.org/0000-0002-9705-2557

Objetivo: compreender, na perspectiva de enfermeiro, sua atuação como líder da equipe de enfermagem na Unidade de Terapia Intensiva. Metodologia: pesquisa qualitativa, com abordagem da fenomenologia social, realizada em um Hospital Público de uma metrópole brasileira, por meio de entrevistas com 11 enfermeiras. Resultados: dificuldades do relacionamento interpessoal provenientes da escassez de pessoal, interferência de outras autoridades hierárquicas e demanda excessiva de trabalho promovem o desgaste nas relações de trabalho e dinâmica da liderança de enfermagem. Melhoria das condições de trabalho da equipe de enfermagem e oportunidade de aperfeiçoamento profissional são mencionadas pelas enfermeiras como expectativas de sua atuação como líderes da Unidade de Terapia Intensiva. Conclusão: políticas de contratação, aperfeiçoamento contínuo dos profissionais e humanização no trabalho, assim como adoção de princípios de liderança que incluam a motivação pessoal, envolvimento e necessidades dos liderados podem contribuir para ampliar a autonomia da enfermeira no serviço, maximizando seu papel de líder.

Descritores: Enfermagem; Liderança; Terapia Intensiva; Pesquisa Qualitativa.

\section{PERFORMANCE OF NURSING LEADERSHIP IN THE INTENSIVE CARE UNIT: A COMPREHENSIVE APPROACH}

Objective: To understand the role of the nursing team leader in the Intensive Care Unit (ICU). Methodology: this was a qualitative study with a social phenomenology approach carried out in a public hospital of a Brazilian metropolitan area. A total of eleven nurses were interviewed. Results: difficulties in interpersonal relationship due to shortage of personnel, interference of other hierarchical authorities, and excessive work demands may lead to conflicting work relationships and affect the ICU nursing leadership. The interviewees pointed out that improvements of the work conditions of the nursing team and the opportunity for professional qualification are some of their expected assignments as ICU leaders. Conclusion: employee hiring policies, continuous improvement of professionals and humanization at work, as well as adoption of leadership principles addressing employee's personal motivation, involvement and needs, can contribute to increase the autonomy of the nurse in the healthcare service and maximize their leadership role.

Descriptors: Nursing; Leadership; Critical Care; Qualitative Research.

\section{ACTUACIÓN DE ENFERMEROS LÍDERES DE UNIDAD DE TERAPIA INTENSIVA: ENFOQUE COMPRENSIVO}

Objetivo: comprender en la perspectiva del enfermero, su actuar como líder del equipo de enfermería en la Unidad de Cuidado Intensivo. Metodología: investigación cualitativa, con abordaje de la fenomenología social, realizada en un Hospital Público de una metrópoli brasileña, por medio de entrevistas con 11 enfermeras. Resultados: dificultades en las relaciones interpersonales provenientes de la escasez de personal, interferencia de otras autoridades jerárquicas, y demanda excesiva de trabajo promueven el desgaste en las relaciones de trabajo y en la dinámica del liderazgo de enfermería. Mejoras de las condiciones de trabajo del equipo de enfermería y oportunidad de crecimiento profesional son mencionadas por las enfermeras como expectativas de su actuar como líderes de la Unidad de Cuidado Intensivo. Conclusión: políticas de contratación, crecimiento continuo de los profesionales y humanización en el trabajo, así como adopción de principios de liderazgo que incluyan la motivación personal, participación y necesidades de los liderados pueden contribuir para ampliar la autonomía de la enfermera en el servicio, maximizando su papel de líder.

Descriptores: Enfermería; Liderazgo; Cuidados Críticos; Investigación Cualitativa. 


\section{INTRODUÇÃO}

A liderança do enfermeiro é fundamental para o trabalho da equipe de enfermagem, pois pode influenciar o comportamento e as atitudes de todos os membros em relação ao cuidado oferecido(1). Estudo, que relacionou liderança de enfermagem e qualidade de cuidados, mostrou que os enfermeiros estabeleciam liderança dialógica e colaborativa com a equipe para desenvolver ações efetivas com vistas a melhorar os cuidados oferecidos. No entanto, as dificuldades experienciadas no direcionamento das ações das pessoas e as características do ambiente hospitalar foram considerados desafios enfrentados no sentido de implementar metas de melhoria da qualidade do atendimento e integração da equipe de enfermagem ${ }^{(2)}$.

O papel de líder requer do enfermeiro visibilidade no âmbito da organização e maior disponibilidade de tempo com a sua equipe, de modo a promover ambiente de trabalho e relações interpessoais saudáveis que valorizem os colaboradores ${ }^{(3)}$.

A Unidade de Terapia Intensiva é um cenário de alta complexidade, dinâmico e especializado em prover cuidados a pacientes graves e instáveis. Contudo, tais particularidades contribuem para o desgaste e sofrimento dos profissionais, favorecendo os conflitos relacionais ${ }^{(4)}$. Considerando a liderança como uma habilidade construída e solidificada a partir de experiências relacionais com pessoas e ambientes, líderes da área de saúde devem buscar a criação de um ambiente de trabalho favorável, harmonioso e menos conflituoso tanto para os profissionais como para os pacientes ${ }^{(5)}$.

A busca na literatura mostrou que a temática liderança em enfermagem envolve aspectos amplos, como gerenciamento dos enfermeiros nas diferentes unidades hospitalares, e quando relacionados à Unidade de Terapia Intensiva, focalizam condições ambientais e fatores relacionados à gestão de modo geral( ${ }^{(4-6)}$, condições nas quais as características do ambiente influenciam na satisfação profissional e nas práticas do cuidado de enfermagem ${ }^{(7-8)}$, nas competências do enfermeiro(9), além do desgaste e insatisfação desse profissional no trabalho ${ }^{(10)}$.

A presente investigação evidencia a percepção do enfermeiro sobre como é liderar a equipe de enfermagem na unidade de terapia intensiva, com destaque para a intersubjetividade vivenciada entre os membros dessa equipe. Indagou-se: como é para o enfermeiro atuar na Unidade de Terapia Intensiva como lider da equipe de enfermagem? Quais suas expectativas em relação à sua atuação?

O objetivo foi compreender, na perspectiva de enfermeiro, sua atuação como líder da equipe de enfermagem na Unidade de Terapia Intensiva.

\section{METODOLOGIA}

\section{Tipo de estudo}

Estudo qualitativo com abordagem da sociologia fenomenológica deAlfred Schütz, que adotou os pressupostos teóricos: intersubjetividade e motivação humana para a ação (motivos porque e para da experiência) ${ }^{(11)}$. Na realidade social, indivíduos apreendem significados que resultam das relações intersubjetivas, que estimulam as ações cotidianas. Essas experiências vividas (passadas e presentes) constituem os motivos porque, que são os conhecimentos adquiridos ao longo da vida, por meio de predecessores e dos advindos da experiência. Esses motivos impulsionam para a idealização de projetos (motivos para). O conjunto de motivos porque e para leva à experiência humana no mundo social(11).

\section{Participantes da pesquisa}

Enfermeiros que atuavam no cargo de líderes de ll Unidades de Terapia Intensiva, independentemente do tempo de formação e atuação no cargo. A amostra foi intencional, sendo que todos participaram da pesquisa, não havendo exclusão de nenhum depoimento.

\section{Local do estudo}

O estudo foi realizado em um Hospital Público de grande portede São Paulo, que possuill Unidades de Terapia Intensiva, separadas por especialidades. Cada unidade é liderada por uma enfermeira e conta com equipe multiprofissional formada por médicos, residentes, enfermeiros, técnicos de enfermagem e fisioterapeutas.

\section{Coleta dos dados}

Realizou-se contato prévio para esclarecimento do objetivo do estudo. Após o aceite, as entrevistas foram agendadas conforme conveniência de cada uma e realizadas nas dependências do estabelecimento hospitalar, em salas reservadas, indicadas pelas próprias entrevistadas. A fim de garantir o anonimato, as enfermeiras foram identificadas pela letra "L" (Líder), seguida do número correspondente à ordem em que as entrevistas foram realizadas ( $\mathrm{Ll}$ a Lll).

Para obtenção dos dados utilizou-se um roteiro de entrevista guiada por questões abertas: como é para você exercer a liderança na Unidade de Terapia Intensiva? Quais são suas expectativas em relação ao seu trabalho? Além disso, registrou-se o tempo de formação e atuação na instituição e no cargo de liderança.

As entrevistas foram realizadas pela pesquisadora principal, no período de janeiro a março de 2017 e gravadas em áudio após permissão dos participantes. O tempo médio de duração de cada uma foi de 55 minutos. 


\section{Procedimento de análise dos dados}

O conteúdo de todos os depoimentos foram considerados pela riqueza de significados, totalizando uma amostra de 11 entrevistas, que foram organizadas, categorizadas e analisadas conforme os passos metodológicos descritos por pesquisadores da fenomenologia social(12). As transcrições foram lidas na íntegra; selecionou-se e agrupou-se os trechos que apresentavam aspectos comuns relacionados aos "motivos porque" e "motivos para" da experiência dos enfermeiros lideres na Unidade de Terapia Intensiva. Discutiram-se os resultados a partir dos conceitos da fenomenologia social de Alfred Schütz e literatura temática.

\section{Procedimentos éticos}

Não houve conflito de interesses na pesquisa e foram levados em consideração os aspectos éticos contidos na Resolução nㅇ 466 de 12 de dezembro de 2012 do Conselho Nacional de Saúde (CNS). As participantes assinaram o Termo de Consentimento Livre e Esclarecido. O projeto foi aprovado pelo comitê de ética do Hospital cenário do estudo, sob o número 1.754.831.

\section{RESULTADOS}

Todas as entrevistadas eram mulheres e especialistas em gerenciamento em enfermagem. A média do tempo de formação foi de 13 anos; 12 anos de atuação na instituição e oito anos no cargo de liderança na Unidade de Terapia Intensiva. Da análise dos depoimentos, emergiu o modo como enfermeiros líderes de enfermagem atuam na Unidade de Terapia Intensiva e suas expectativas em relação à sua atuação.

Atuação de enfermeiras líderes de enfermagem na Unidade de Terapia Intensiva (motivo porque)

Os depoimentos das enfermeiras mostram que é preciso ter atitudes positivas para conseguir resolver os conflitos advindos das relações humanas e liderar a equipe de enfermagem: “...o que faz a diferença no meu trabalho é o trato com as pessoas, é a forma como lido com minha equipe ...ao liderar minha equipe, não posso me pautar somente nos textos teóricos, eles são maravilhosos, mas na prática é bem diferente ...algumas situações são muito conflituosas e dificeis e eu tenho que me posicionar para motivá-los a mudar e melhorar o que não está bom" (L1O).

A demanda excessiva de trabalho na Unidade de Terapia Intensiva e a interferência de outras autoridades hierárquicas são alguns dos fatores mencionados pelas enfermeiras como dificultadores do relacionamento interpessoal no serviço, distanciando-as dos membros da equipe de enfermagem: “...a demanda é grande ...fora o desgaste físico e emocional, ...me sinto o recheio do sanduíche, fico no meio sendo bombardeada pelos dois lados ...e por causa disso não consigo trabalhar melhor com minha equipe" (Ll); “...sou cobrada pelos meus diretores... a quantidade de material e recursos humanos é ruim, e isso impacta na minha forma de liderar" (L5); "... ter que liderar as pessoas é muito difícil” (L4); “...por mais que eu fale... a maior influência não é minha, mas sim dos meus superiores hierárquicos" (L3).

A escassez de recursos humanos que sobrecarrega os profissionais foi mencionada pelas enfermeiras como dificultador do trabalho de liderança na Unidade de Terapia Intensiva: “...as pessoas estão ficando sobrecarregadas ...essa sobrecarga impede a troca de informações e o estabelecimento de boa relação, porque estão sempre no limite ...para mim é mais complicado ainda, porque tenho que tentar apoiar e ajudar minha equipe a alcançar os objetivos ...mas é difícil, porque nem eles e nem eu temos tempo para discutirmos os problemas e, com isso, sinto que estou falhando na minha função de líder" (L7).

As restrições médicas dos trabalhadores diminuem o quantitativo de pessoal, gerando mais conflitos no ambiente de trabalho: “...outro problema são os funcionários com restrições ...é difícil trabalhar essa questão ...fazer com que os demais compreendam que precisam desenvolver o trabalho em equipe e serem mais tolerantes é muito difícil" (L3).

Apesar das dificuldades enfrentadas as enfermeiras demonstram satisfação com seu papel de líder e se esforçam para desempenhá-lo bem: “...mesmo que a carga horária seja excessiva e as condições de trabalho difíceis, eu desenvolvo a liderança da minha equipe da melhor forma possivel dentro da realidade em que me encontro e com os recursos que estão disponiveis" (L1O).

Promover o envolvimento dos membros da equipe na resolução dos problemas foi uma estratégia relatada pelas participantes para maximizar a liderança: “...a instituição não é perfeita e tem seus problemas, mas eu procuro dar "feedback" para a minha equipe. Sempre que possivel, mostro o que deu e o que não deu certo e peço a opinião deles sobre como podemos melhorar. Procuro valorizar minha equipe" (LIl).

Expectativas das enfermeiras líderes na Unidade de Terapia Intensiva (motivos para)

A valorização profissional foi referida como sendo uma expectativa das enfermeiras que atuam como líderes: “...espero ter mais valorização profissional para poder desempenhar meu papel de liderança” (LI); “...as pessoas precisam reconhecer o meu trabalho, o meu mérito como lider" (L2).

Como um modo de valorização profissional, as enfermeiras esperam conseguir um local adequado para as pausas no trabalho e legalização do horário de descanso. Isso 
foi mencionado como um fator facilitador de liderança da equipe: “...tem que ter uma copa e um banheiro decente ...outra coisa importante é a legalização do horário de descanso do pessoal do noturno, porque sabemos que o descanso existe, mas fica tudo subentendido. Só assim conseguirei estimular e incentivar minha equipe a dar o melhor de si" (L4); ... sonho com um plano de carreira que me possibilite trabalhar e desenvolver melhor a minha equipe (L8).

As entrevistadas referem também o desejo de aperfeiçoamento profissional da equipe de enfermagem: “...espero que haja mais treinamento para as enfermeiras assistenciais. ... percebo que nem sempre as decisões são coesas e isso dificulta a minha liderança com a equipe (L6); “... gostaria que houvesse um plano de aprendizagem contínua para os enfermeiros para que conseguíssemos adequar teoria e prática" (L9)

\section{DISCUSSÃO}

Na perspectiva das enfermeiras, a liderança na Unidade de Terapia Intensiva é permeada por conflitos relacionais com a equipe de enfermagem, além de sofrer interferência de superiores hierárquicos, o que impacta diretamente nas relações e dinâmica do trabalho. Uma pesquisa realizada na Austrália mostrou que a colaboração, em todos os níveis de hierarquia, e a comunicação positiva na Unidade de Terapia Intensiva facilitaram o atendimento aos pacientes e as tomadas de decisões. Opostamente, os conflitos entre os membros da equipe, as limitações ambientais e a falta de um espaço reservado para discussão dos casos foram os principais causadores de frustração e empecilhos para colocar em prática os planos de cuidado(13).

Sabe-se que a competência de liderança requer autonomia nas tomadas de decisões. Estudo que associou ambiente das práticas de enfermagem e satisfação profissional em Unidades de Terapia Intensiva mostrou que o ambiente foi favorável para a autonomia e relações entre médicos e enfermeiros, apontando satisfação profissional por 272 (94,77\%) participantes ${ }^{(8)}$. Na China, investigação que avaliou o desgaste do enfermeiro e a insatisfação no trabalho em unidades críticas, também concluiu que a satisfação profissional e o bom desenvolvimento da liderança estão especialmente ligados à autonomia e ao bom relacionamento da equipe multiprofissional( ${ }^{(10)}$.

De acordo com as entrevistadas, a escassez de recursos humanos, que inclui os casos de restrição médica dos membros da equipe de enfermagem, impactaram diretamente na sobrecarga dos profissionais sob a liderança do enfermeiro. Estudo mostrou que limites organizacionais que incluem vínculos precários de trabalho, grande rotatividade de pessoal, desvalorização e ausência de boas condições ambientais são algumas das causas de desmotivação e 44 insatisfação dos profissionais de enfermagem na Unidade de
Terapia Intensiva(14)

Ainda queas enfermeiras desteestudotenham evidenciado diferentes problemas ao liderar sua equipe na Unidade de Terapia Intensiva, procuraram envolver os membros na resolução dos problemas e fornecer "feedback" sobre as decisões tomadas e resultados obtidos, o que demonstrou o enfrentamento das adversidades e a valorização da equipe de enfermagem. É imprescindivel investir no relacionamento interpessoal e envolver os liderados no processo decisório para obter resolubilidade dos problemas e alcançar as mudanças atitudinais, necessárias para o desenvolvimento da assistência prestada ${ }^{(15)}$

O fortalecimento e entrosamento dos grupos de trabalho de modo a valorizar os talentos individuais e diluir o poder na equipe podem promover o crescimento e corresponsabilização de cada membro. Contudo, esse processo somente é possivel de ser alcançado por meio da interação entre os envolvidos ao longo do tempo(16). Pesquisa que refletiu sobre a questão da liderança em enfermagem a partir do referencial sistêmico de Frontier, mostrou não ser possivel liderar sozinho, assim como não é possivel cuidar sozinho. A liderança e o cuidado precisam estar entrelaçados em uma teia de relações de modo a promover harmonia e equilibrio nas interrelações, favorecendo um constante aperfeiçoamento das relações e do cuidado(5).

Liderar centrado nas relações pessoais contribui para a melhoria da produtividade e dos resultados no ambiente de trabalho, especialmente na Unidade de Terapia Intensiva. Nesse sentido, as teorias contemporâneas sobre liderança têm sido implementadas de modo a valorizar o alto grau de motivação do pessoal, por meio de uma relação baseada na confiança, incentivo à criatividade e inovação, com atenção para as necessidades dos liderados (líder transformacional); envolvimento dos liderados nas decisões, aumentando a confiança, o engajamento e a satisfação no trabalho (líder autêntico) e observando o comportamento dos integrantes do grupo para implementar ações para o alcance dos objetivos (liderança situacional)(15)

O ambiente da Unidade de Terapia Intensiva descrito pelas entrevistadas mostra a influência dessa realidade social sobre a atuação dos enfermeiros líderes. De acordo com a fenomenologia social, as pessoas se interrelacionam no contexto social, o que interfere no seu modo de agir(11) A atuação de enfermeiros líderes na Unidade de Terapia Intensiva move esses profissionais a esboçarem expectativas em relação à liderança. Essas expectativas são descritas por Schütz como sendo os motivos que impulsionam à ação social (motivos para). As enfermeiras entrevistadas almejam investimento na melhoria das condições de trabalho da equipe de enfermagem e oportunidade de aperfeiçoamento profissional para que possam desenvolver a contento o papel 
de líderes.

Pesquisa realizada no Texas (EUA) mostrou que a valorização profissional, a comunicação efetiva, o contingente adequado de recursos humanos, entre outros, são essenciais para atuação de enfermeiras líderes de terapia intensiva e, consequentemente, para a manutenção do ambiente saudável ${ }^{(17)}$. Algumas medidas por parte da instituição de saúde são imprescindiveis para que os enfermeiros se sintam valorizados. Dentre elas, destaca-se a disponibilização de um local adequado para pausas no trabalho e a normatização do horário de descanso noturno. O trabalho no período noturno no hospital, aliado às normas contratuais não favoráveis, são alguns dos fatores que contribuem para a insatisfação dos profissionais. Alterar a rotina de vida, e especialmente o sono, pode gerar maior desgaste físico, redução da capacidade cognitiva e da execução de atividades, favorecendo a ocorrência de doenças e acidentes relacionados ao trabalho ${ }^{(18)}$.

O desejo de aperfeiçoamento profissional foi referido pelas enfermeiras líderes da Unidade de Terapia Intensiva. Estudo de revisão apontou a relevância da capacitação profissional nesse setor, dada à necessidade do uso de tecnologias emergentes e de estratégias eficazes para a assistência de qualidade. Por isso, a busca pelo aperfeiçoamento deve acontecer de forma natural e contínua ${ }^{(19)}$.

O encontro com as experiências passadas e presentes dos indivíduos, somados às suas expectativas, podem mudar a realidade vivenciada no ambiente da Unidade de Terapia Intensiva. Para Schütz, a compreensão intersubjetiva permite a interrelação no mundo social(11). É nesse mundo que as ações da equipe de enfermagem se consolidam, e onde esta equipe pode intervir e propor novas formas de atuação. $\mathrm{Na}$ realidade social, as pessoas se interrelacionam, conhecem umas às outras compartilhando o mesmo tempo, espaço e situação social(11).

\section{Limitações do Estudo}

Esta investigação traz como limitação o fato de ter sido realizada com um grupo social de uma instituição pública específica, no entanto, permite avançar no conhecimento e contribuir para o embasamento de melhores práticas em relação ao exercício profissional de enfermeiros líderes que atuam em Unidades de Terapia Intensiva.

\section{Contribuições do estudo para a prática}

Os resultados deste estudo despertam para questões que necessitam de investimentos institucionais, visando maximizar a atuação do enfermeiro líder de enfermagem na Unidade de Terapia Intensiva. Políticas de contratação, aperfeiçoamento contínuo dos profissionais e humanização no trabalho, assim como a adoção de princípios de liderança que incluam motivação pessoal, envolvimento e necessidades dos liderados podem contribuir para ampliar a autonomia da enfermeira no serviço, maximizando seu papel de líder.

\section{CONCLUSÃO}

Este estudo permitiu compreender que as dificuldades do relacionamento interpessoal provenientes da escassez de pessoal, interferência de outras autoridades hierárquicas e demanda excessiva de trabalho promovem o desgaste nas relações de trabalho e dinâmica da liderança de enfermagem. Melhoria das condições de trabalho da equipe de enfermagem e oportunidade de aperfeiçoamento profissional são mencionadas pelas enfermeiras como expectativas de sua atuação como líderes da Unidade de Terapia Intensiva.

Dada à importância do contexto social no exercício da liderança, recomenda-se realização de pesquisas sobre a liderança de enfermeiros em outras Unidades de Terapia Intensiva, para que se possa ampliar o conhecimento sobre as experiências vivenciadas nesses serviços, além de instrumentalizar a gestão para converter a teoria em prática factivel de ser aplicada.

\section{Contribuição dos autores}

Concepção e desenho, análise e interpretação dos dados, redação do artigo, revisão crítica, revisão final: Claudete Aparecida Conz; Reginaldo Santos de Aguiar; Heliandra Holanda Reis; Maria Cristina de Jesus Pinto; Vera Lúcia Mira; Miriam Aparecida Barbosa Merighi. 


\section{REFERÊNCIAS}

1- Pereira FM. Sintomas depressivos no puerpério: uma revisão de literatura [Internet]. São Paulo: Marilia; 2011. Disponivel em: http:// pesquisa.bvsalud.org/bvsvs/resource/pt/ses-31110

2- Valença CN, Germano RM. Prevenindo a depressão puerperal na estratégia saúde da familia: Ações do enfermeiro no pré-natal. Rev Rene. Fortaleza. 2010; 11(2):129-39. Disponivel em: http://periodicos.ufc.br/ rene/article/view/4541. Acesso em 13 de agosto de 2019.

3- Barbosa RCA. Prevalência e Incidência de Depressão Pós-Parto e Sua Associação com o Apoio Social [dissertação]. Recife, PE: Universidade Federal de Pernambuco; 2008. Disponivel em: https://repositorio. ufpe.br/bitstream/123456789/9231/1/arquivo4156_1.pdf

4- Moraes GPA, Lorenzo L, Pontes GAR, Montenegro MC, Cantilino A. Screening and diagnosing postpartum depression: when and how? Trends Psychiatry Psychother. 2017:39(1) - 54-61. Disponivel em: http://www.scielo.br/scielo.php?script=sci_arttext\&pi$d=$ S2237-60892017000100054

5- Theme Filha MM, Ayers S, Gama SGN, Leal MC. Factors associated with postpartum depressive symptomatology in Brazil: The Birth in Brazil National Research Study, 2011/2012. J Affect Disord. 2016; 194:159-67. Disponivel em: https://www.sciencedirect.com/science/ article/abs/pii/S0165032715306789?via\%3Dihub Acesso em 30 de maio de 2019.

6- Kingston D, Kehler H, Austin MP, Mughal MK, Wajid A, Vermeyden L, et al. Trajectories of maternal depressive symptoms during pregnancy and the first 12 months postpartum and child externalizing and internalizing behavior at three years. PLoS ONE. $2018 \mathrm{Apr}$ 13; (4). Disponivel em: https://journals.plos.org/plosone/article?id=10.1371/journal. pone.0195365

7- Brasil. Conselho Nacional de Educação. Câmara de Educação Superior. Resolução no 3/2001. Diário Oficial da União, Brasilia. 2001 Nov 9 Sect 1: 37. Disponivel em: <http://portal.mec.gov.br/cne/arquivos/pdf/ CESO3.pdf> Acesso em 3 de dezembro de 2018

8- Schardosim J M, Heldt E. Escalas de rastreamento para depressão pós-parto: uma revisão sistemática. Rev Gaúcha Enferm. (Online). Porto Alegre. 2011 Mar; 32(1):159-66. Disponivel em: <http://www.scielo.br/scielo.php?script=sci_arttextEpid=S1983-14472011000100021\&lng=enEnrm=iso>

9- Cox JL, Holden JM, Sagovsky R. Detection of postnatal depression: development of the 10-item Edinburgh Postnatal Depression Scale. B J Psychiatry. 1987; 150:782-86. Disponivel em: <https://www.cambridge.org/core/journals/the-british-journal-of-psychiatry/article/detection-of-postnatal-depression/E18BC62858DBF2640C33DCC8B572F02A>Acesso em 17 de junho de 2019

10- Santos IS, Matijasevich A, Tavares BF, Barros AJD, Botelho IP. Lapolli C, et al. Validation of the Edinburgh Postnatal Depression Scale
(EPDS) in a sample of mothers from the 2004 Pelotas Birth Cohort Study. Cad Saúde Pública. Rio de Janeiro. 2007; 23(11):2577-88. Disponivel em:

12- Brasil. Ministério da Saúde. Conselho Nacional de Saúde. Resolução no 466/2012. Diário Oficial da União. Brasilia. 2012 Dez 12. Disponivel em: <http://bvsms.saude.gov.br/bvs/saudelegis/cns/2013/ res0466_12_12_2012.html> Acesso em 23 de junho de 2019.

13- Hartmann JM. Sassi RAM, Cesar JA. Depressão entre puérperas: prevalência e fatores associados. Cad Saúde Pública. 2017 33(9):e00094016. Disponivel em: <http://www.scielo.br/pdf/csp/ v33n9/1678-4464-csp-33-09-e00094016.pdf> Acesso em 30 de maio de 2019.

14. Araújo IS, Aquino KS, Fagundes LKA, Santos VC. Postpartum Depression: Epidemiological Clinical Prole of Patients Attended In a Reference Public Maternity in Salvador-BA. Rev Bras Ginecol Obstet. 2019:41(3):155-63

15- Figueira P, Corrêa H, Malloy-Diniz L, Romano- Silva MA. Escala de depressão pós-natal de Edimburgo para triagem no sistema público de saúde. Rev Saúde Pública. 2009; 43 Suppl 1:79-84. Disponivel em: <http://www.scielo.br/scielo.php?script=sci_arttext\&pid=S0034-89102009000800012>. Acesso em 18 de junho de 2019.

16- Buist A, Janson H. Childhood sexual abuse, parenting and postpartum depression: A 3-year follow-up study. Child Abuse Negl. 2001; 25 909-21. Disponivel em: <https://www.sciencedirect.com/science/article/abs/pii/S0145213401002460?via\%3Dihub> Acesso em 18 de junho de 2019.

17- Melo SB, Jordão RRR, Guimarães FJ, Perrelli JGA, Cantilino A, Sougey EB. Sintomas depressivos em puérperas atendidas em Unidades de Saúde da Familia. Rev Bras Saúde Mater Infant. Recife. Jan-mar 2018; 18(1): 171-77. Disponivel em: <http://www.scielo.br/scielo.php?script=sci_arttext\&pid=S1519=38292018000100163-\&lng=en\&nrm=iso\&tlngpt> Acesso em 24 de junho de 2019.

18- Morais MLS, Fonseca LAM, David VF, Viegas LM, Otta E. Fatores psicossociais e sociodemográficos associados à depressão pós-parto: Um estudo em hospitais público e privado da cidade de São Paulo. Brasil. Estud Psicol (Natal). 2015 Jan-Mar; 20(1): 40-9. Disponivel em: http://dx.doi.org/10.5935/1678-4669.20150006 Acesso em 3 de julho de 2019 .

19- Félix TA, Ferreira AGN, Siqueira DA, Nascimento KV, Ximenes Neto FRG, Mira OL. Atuação da enfermagem frente à depressão pós-parto nas consultas de puericulturas. Rev Enfermeria Global. 2013 Jan; (29): 420-35. Disponivel em: <http://scielo.isciii.es/pdf/eg/v12n29/pt_enfermerial.pdf > Acesso em 12 de agosto de 2019 